\title{
Pneumonectomy for Lung Cancer Treatment in The Netherlands: Between-Hospital Variation and Outcomes
}

\author{
Naomi Beck ${ }^{1,2}$ (1) - Thomas J. van Brakel ${ }^{3} \cdot$ Hans J. M. Smit ${ }^{4}$ David van Klaveren ${ }^{5}$ • \\ Michel W. J. M. Wouters ${ }^{2,6}$ - Wilhelmina H. Schreurs ${ }^{7}$
}

Published online: 23 September 2019

(C) The Author(s) 2019

\begin{abstract}
Background Pneumonectomy in lung cancer treatment is associated with considerable morbidity and mortality. Its use is reserved only for patients in whom a complete oncological resection by (sleeve) lobectomy is not possible. It is unclear whether a patients' risk of receiving a pneumonectomy is equally distributed. This study examined betweenhospital variation of pneumonectomy use for primary lung cancer in the Netherlands.

Methods Data from the Dutch Lung Cancer Audit for Surgery from 2012 to 2016 were used to study the use of pneumonectomy for primary lung cancer in the Netherlands. Using multivariable logistic regression, factors associated with pneumonectomy use were identified and the expected number of pneumonectomies per hospital was determined. Subsequently, the observed/expected ratio ( $O / E$ ratio) per hospital was calculated to study betweenhospital differences.

Results Of the 8446 included patients, 659 (7.8\%) underwent a pneumonectomy with a mean postoperative mortality of $7.1 \%(n=47)$. Factors associated with receiving a pneumonectomy were age, gender, cardiac and pulmonary comorbidities, tumor side, size and histopathology. The pneumonectomy use in the Netherlands varied considerably between hospitals (IQR 5.5-10.1\%). Three hospitals out of 51 performed significantly less pneumonectomies than expected $(O / E$ ratio $<0.5)$ and three significantly more $(O / E$ ratio $>1.7)$. In the latter group, severe complications were more frequent, taking other influencing factors into account (OR 1.51, 95\% CI 1.05-2.19).

Conclusions There is a considerable between-hospital variation in pneumonectomy use in lung cancer treatment. To further optimize surgical lung cancer care, we suggest center-specific feedback on pneumonectomy use and the development of a risk-adjusted pneumonectomy indicator.
\end{abstract}

Electronic supplementary material The online version of this article (https://doi.org/10.1007/s00268-019-05190-w) contains supplementary material, which is available to authorized users.

Naomi Beck

n.beck@lumc.nl

1 Department of Surgery, Leiden University Medical Center, Albinusdreef 2, 2333 ZA Leiden, The Netherlands

2 Dutch Institute for Clinical Auditing, Rijnsburgerweg 10, 2333 AA Leiden, The Netherlands

3 Department of Cardiothoracic Surgery, Leiden University Medical Center, Albinusdreef 2, 2333 ZA Leiden, The Netherlands
4 Department of Pulmonology, Rijnstate, Wagnerlaan 55, 6815 AD Arnhem, The Netherlands

5 Medical Statistics, Department of Biomedical Data Sciences, Leiden University Medical Center, Einthovenweg 20, 2333 ZC Leiden, The Netherlands

6 Department of Surgical Oncology, The Netherlands Cancer Institute/Antoni van Leeuwenhoek, Plesmanlaan 121, 1066 CX Amsterdam, The Netherlands

7 Department of Surgery, North-West Clinics, Wilhelminalaan 12, 1815 JD Alkmaar, The Netherlands 


\section{Introduction}

Anatomical parenchymal resection is the cornerstone in curative treatment for primary lung cancer. In certain cases, a complete oncologic resection cannot be obtained by a (sleeve) lobectomy and a pneumonectomy is considered the resection of choice. However, pneumonectomy is associated with considerable postoperative morbidity and mortality compared to less extensive resections and is an individual predictor of these negative outcomes [1-5]. Reduction in adverse outcomes of lung surgery may therefore be achieved by decreasing the number of pneumonectomies.

Several nationwide registries reported on the national pneumonectomy use [6-12]. Although the optimal target proportion of pneumonectomies is unclear and partly depends on casemix, differences in the threshold at which a pneumonectomy is performed may identify improvement potential. As suggested by Jakobsen et al. [6], the proportion of pneumonectomies could eventually function as a quality indicator in surgical lung cancer care.

To apply such a quality indicator, between-hospital variation in pneumonectomy use and possibilities for proper casemix adjustment first need to be studied. Therefore, the aim of this study was to investigate between-hospital variation in the use and outcomes of pneumonectomies for primary lung cancer in the Netherlands and to identify factors associated with pneumonectomy use.

\section{Methods}

\section{Data source and study population}

Data were derived from the Dutch Lung Cancer Audit for Surgery (DLCA-S) [13]. The DLCA-S is a nationwide mandatory registry including all patients undergoing surgery for lung cancer and is part of the multidisciplinary Dutch Lung Cancer Audit (DLCA), in which all major treatment disciplines evaluate care [13].

In the DLCA-S, data are collected on hospital level. Distinction on individual surgeon level is not possible. In cases of a surgeon operating in different hospitals, the procedure is attributed to the hospital where it was performed. Collected data include information on patient and tumor characteristics, diagnostic workup (e.g., discussion in a multidisciplinary meeting), surgical procedure, postoperative outcomes and pathology [14]. Independent onsite data verification processes are used to ensure data quality [15]. From 2015 on, there is a 100\% coverage of NSCLC resection registration [14].
For this study, no ethical approval or informed consent was required under Dutch law.

Patients with a primary lung cancer resection between January 2012 and December 2016 were included. Minimum data registry criteria to be eligible for analyses included: age, gender, operation date, type of surgery, tumor side, postoperative histology and vital status 30 days after surgery and/or at time of discharge.

Wedge excisions were excluded for the analysis of anatomical resections, since these are considered oncologically insufficient. Primary lung cancer comprised a postoperative histology of: adenocarcinoma, squamous cell carcinoma, carcinoid, large cell carcinoma, small cell carcinoma and non-small cell carcinoma not otherwise specified.

\section{Population characteristics and main outcomes}

Analyzed patient characteristics were: age at time of surgery, gender, lung function, ECOG performance status and ASA classification. Preoperative lung function was analyzed as a composite measure of FEV1\% (forced expiratory volume in $1 \mathrm{~s}$ percentage of normal) and DLCO\% (diffusing lung capacity for oxygen percentage of normal), in three categories: FEV1\% and DLCO $\% \geq 80 \%$ or one of the values not registered (1), FEV1\% or DLCO $\%<80 \%$ (2) and both FEV1\% and DLCO\% not registered (3). These cutoff values are in accordance with the evidence-based Dutch guideline [16, 17].

Disease characteristics and pre-surgical treatment characteristics were: tumor side, induction therapy (none/unknown, chemotherapy, radiotherapy and chemoradiotherapy), tumor stage (according to the seventh edition of the TNM staging system) and postoperative histology.

The outcomes assessed were postoperative mortality and postoperative complicated course. Postoperative mortality was defined as mortality within 30 days after surgery or during the primary hospital admission. Postoperative complicated course was defined as any complication leading to prolonged hospital stay ( $\geq 14$ days) and unplanned re-intervention or mortality and was used to reflect only severe complications.

\section{Between-hospital variation in applying pneumonectomy}

Between-hospital variation in applying pneumonectomy was studied by comparing the observed with the expected number of pneumonectomies per hospital. With a multivariable logistic regression model, after controlling for collinearity, patient and tumor characteristics associated with the risk of undergoing pneumonectomy were 
identified. Discriminative ability of the model was assessed by area under the ROC curve (AUC).

Subsequently, by using the coefficients of this multivariable model the expected 'pneumonectomy risk' per patient was calculated, which in turn was used to calculate the expected number of pneumonectomies on hospital level. Then, by dividing the number of observed by the number of expected pneumonectomies per hospital, the observed/expected ratio ( $O / E$ ratio) was calculated per hospital. An $O / E$ ratio $>1$ indicates that the hospital performed more pneumonectomies than expected based on the hospital population, whereas an $O / E$ ratio $<1$ indicates a lower pneumonectomy use than expected. Between-hospital variation in $O / E$ ratio was displayed using a funnel plot, with $95 \%$ confidence intervals (95\% CI) [18].

Hospital characteristics (e.g., case volume or type of hospital) were not included in the model since the chance of undergoing a pneumonectomy or a different resection type should not depend on that. If these factors would be included in the model, the effect can be distorted or even nullified [19].

\section{Pneumonectomy/sleeve resection ratio}

From 2015 on, the DLCA-S contains information on sleeve resections. Hypothesizing that sleeve lobectomies and pneumonectomies are performed in similar patient populations, a ratio between these two operation types could demonstrate differences in preference of indication per hospital.

Statistical significance was set at a threshold of 0.05 , with P-values calculated by two-sided tests.

Statistical analyses were performed using SPSS (IBM SPSS Statistics for Macintosh, version 23.0).

\section{Results}

\section{Population characteristics}

A total of 8446 patients underwent a primary lung cancer resection and were eligible for analyses. Of them, 659 (7.8\%) underwent pneumonectomy, $7226 \quad(85.6 \%)$ (bi)lobectomy or anatomical segment resection and 561 $(6.6 \%)$ wedge excision.

After excluding the wedge excisions, 7885 patients with an anatomical resection remained, of whom $8.4 \%$ (659) underwent pneumonectomy. These 7885 patients were divided over 51 hospitals, with a mean number of patients per hospital of 155, SD 97, range: 8-377.

Of patients with an anatomical resection, mean age was 66 years, $55.7 \%$ was male $(n=4395), 76.6 \%$ had an ECOG performance score $0-1(n=6040), 70.9 \%$ had an
ASA score I-II $(n=5587), 6.3 \%$ received induction therapy $(n=498), 43.1 \%$ had a left-sided tumor $(n=3399)$, $81.5 \%$ had a pathological stage $\leq$ II $(n=6421)$ and $55.2 \%$ had an adenocarcinoma $(n=4352)$.

Table 1 displays the characteristics of all patients undergoing pneumonectomy. Compared to the total anatomical resection group, pneumonectomies were performed in slightly younger patients, more often of male sex, in more advanced disease stages, left-sided tumors and squamous cell carcinomas.

Of all anatomical resection, 5.3\% $(n=417)$ was performed in hospitals with less than 20 resections a year (low volume considering the minimum annual volume standards set by the Association of Surgeons in the Netherlands), $56.0 \%(n=4416)$ in hospitals with 20 to 49 resections a year and $38.7 \%(n=3052)$ in hospitals with 50 or more resections a year. The pneumonectomies in these three hospital volume categories were, respectively, 8.9\%, 7.9\% and $8.9 \%$.

\section{Factors associated with pneumonectomy}

Age, gender, cardiac and pulmonary comorbidities, tumor side, clinical tumor stage (cT) and histopathology are individual factors significantly associated with receiving a pneumonectomy (Table 2). The discriminative ability of a multivariable model with these factors was fairly good (AUC), 0.80, 95\% CI 0.78-0.82.

Supplementary Fig. 1 visually demonstrates the association between cT and histopathology and the pneumonectomy proportion.

\section{Between-hospital variation}

The use of pneumonectomy as an anatomical resection for primary lung cancer per hospital ranged from 0.0 to $25.3 \%$ (national mean 8.4\%). Fifty per cent of hospitals (interquartile range-IQR) performed a pneumonectomy in $5.5-10.1 \%$ of their patients.

Between-hospital variation remained after adjustment for relevant factors (Fig. 1). Out of 51 hospitals, three performed significantly more pneumonectomies than expected $(O / E$ ratio $>1.7)$. Three performed significantly less pneumonectomies than expected, with an $O / E$ ratio $<0.5$; the percentage of pneumonectomies performed is $>50$ less than expected. All six hospitals were middlesized non-academic centers.

After adjustment for relevant factors [5], there were no significant differences in postoperative mortality and complicated course after a pneumonectomy performed in the three hospitals with more pneumonectomies (71 patients included) compared to the three hospitals with less pneumonectomies (19 patients included) than expected 
Table 1 Population characteristics and postoperative outcomes of patients with primary lung cancer undergoing an anatomical parenchymal resection, stratified for resection type

\begin{tabular}{|c|c|c|c|c|c|}
\hline & \multicolumn{2}{|c|}{ Pneumonectomy } & \multicolumn{2}{|c|}{ (Bi)lobectomy and segmentectomy } & \multirow[t]{2}{*}{$p$} \\
\hline & $N$ & $\%$ & $N$ & $\%$ & \\
\hline Of total anatomical parenchymal resections & 659 & 8.4 & 7226 & 91.6 & - \\
\hline Gender & & & & & $<0.001$ \\
\hline Male & 452 & 68.6 & 3943 & 54.6 & \\
\hline Female & 207 & 31.4 & 3283 & 45.4 & \\
\hline Age mean [median] $( \pm \mathrm{SD})$ & $65[6$ & & $66[67$ & & \\
\hline Age (years) & & & & & $<0.001$ \\
\hline$<60$ & 143 & 21.7 & 1681 & 23.3 & \\
\hline $60-64$ & 144 & 21.9 & 1256 & 17.4 & \\
\hline $65-69$ & 143 & 21.7 & 1491 & 20.6 & \\
\hline $70-74$ & 145 & 22.0 & 1433 & 19.8 & \\
\hline $75+$ & 84 & 12.7 & 1365 & 18.9 & \\
\hline \multicolumn{6}{|l|}{ Lung function } \\
\hline FEV $1 \%$ and DLCO $\% \geq 80 \%$ & 173 & 26.3 & 2358 & 32.7 & 0.004 \\
\hline FEV $1 \%$ or DLCO $\%<80 \%$ & 443 & 67.2 & 4439 & 61.4 & \\
\hline Unknown & 43 & 6.5 & 429 & 5.9 & \\
\hline Performance score* & & & & & 0.063 \\
\hline$<2$ & 489 & 74.2 & 5551 & 76.8 & \\
\hline$\geq 2$ & 35 & 5.3 & 260 & 3.6 & \\
\hline Unknown & 135 & 20.5 & 1415 & 19.6 & \\
\hline ASA score & & & & & 0.323 \\
\hline $\mathrm{I}-\mathrm{II}$ & 451 & 68.4 & 5136 & 71.1 & \\
\hline $\mathrm{III}+$ & 186 & 28.2 & 1847 & 25.5 & \\
\hline Unknown & 22 & 3.4 & 243 & 3.4 & \\
\hline Side & & & & & 0.001 \\
\hline Left & 408 & 61.9 & 2991 & 41.4 & \\
\hline Right & 251 & 38.1 & 4235 & 58.6 & \\
\hline Induction therapy & & & & & $<0.001$ \\
\hline No & 589 & 89.3 & 6798 & 94.0 & \\
\hline Chemoradiotherapy & 31 & 4.7 & 265 & 3.7 & \\
\hline Chemotherapy & 1 & 0.2 & 137 & 1.9 & \\
\hline Radiotherapy & 38 & 5.8 & 26 & 0.4 & \\
\hline Pathological stage & & & & & $<0.001$ \\
\hline Stage I and occult & 107 & 16.2 & 4155 & 57.5 & \\
\hline Stage II & 266 & 40.4 & 1893 & 26.2 & \\
\hline Stage III+ & 255 & 38.7 & 939 & 13.0 & \\
\hline Unknown & 31 & 4.7 & 239 & 3.3 & \\
\hline Pathological $\mathrm{T}$ stage & & & & & $<0.001$ \\
\hline T1 (T0, Tis, Tx) & 89 & 13.5 & 3087 & 42.7 & \\
\hline $\mathrm{T} 2$ & 274 & 41.6 & 2915 & 40.3 & \\
\hline $\mathrm{T} 3$ & 214 & 32.5 & 1048 & 14.5 & \\
\hline $\mathrm{T} 4$ & 80 & 12.1 & 152 & 2.2 & \\
\hline Unknown & 2 & 0.3 & 24 & 0.3 & \\
\hline Postoperative histology & & & & & $<0.001$ \\
\hline Adenocarcinoma & 189 & 28.7 & 4163 & 57.6 & \\
\hline Squamous cell & 410 & 62.2 & 2194 & 30.4 & \\
\hline Different** & 60 & 9.1 & 869 & 12.0 & \\
\hline Postoperative mortality*** & 47 & 7.1 & 123 & 1.7 & \\
\hline
\end{tabular}

*Performance score using ECOG/WHO. **Different: SCLC, carcinoid, adenosquamous, large cell (NET) and not otherwise specified. ***Defined as postoperative 30-day or in-hospital mortality 
Table 2 Factors associated with receiving a pneumonectomy. Number of patients included: $N=7885$

\begin{tabular}{|c|c|c|c|c|}
\hline & \multicolumn{4}{|c|}{ Resection using pneumonectomy } \\
\hline & \multicolumn{2}{|c|}{ Unadjusted* } & \multicolumn{2}{|c|}{ Adjusted** } \\
\hline & $\mathrm{OR}^{\wedge}$ & $95 \% \mathrm{CI}^{\wedge}$ & $\mathrm{OR}^{\wedge}$ & $95 \% \mathrm{CI}^{\wedge}$ \\
\hline \multicolumn{5}{|l|}{ Age } \\
\hline$<60$ & Ref & & Ref & \\
\hline $60-64$ & 1.35 & $1.06-1.72$ & 1.16 & $0.89-1.50$ \\
\hline $65-69$ & 1.13 & $0.89-1.44$ & 0.88 & $0.67-1.14$ \\
\hline $70-74$ & 1.19 & $0.93-1.51$ & 0.81 & $0.62-1.06$ \\
\hline $75+$ & 0.72 & $0.55-0.96$ & 0.47 & $0.34-0.64$ \\
\hline \multicolumn{5}{|l|}{ Gender } \\
\hline Male & Ref & & Ref & \\
\hline Female & 0.55 & $0.46-0.65$ & 0.72 & $0.60-0.87$ \\
\hline \multicolumn{5}{|l|}{ Cardiac comorbidity } \\
\hline No & Ref & & Ref & \\
\hline Yes & 0.74 & $0.61-0.90$ & 0.75 & $0.60-0.92$ \\
\hline \multicolumn{5}{|l|}{ Pulmonary comorbidity } \\
\hline No & Ref & & Ref & \\
\hline Yes & 0.75 & $0.63-0.89$ & 0.69 & $0.57-0.83$ \\
\hline \multicolumn{5}{|l|}{ Lung function } \\
\hline $\mathrm{FEV}^{\mathrm{a}}$ or $\mathrm{DLCO}^{\mathrm{b}} \geq 80 \%$ & Ref & & NA & \\
\hline FEV1 and DLCO $<80 \%$ & 1.36 & $1.13-1.63$ & NA & \\
\hline FEV1 and DLCO unknown & 1.37 & $0.96-1.94$ & NA & \\
\hline \multicolumn{5}{|l|}{ Side of tumor } \\
\hline Left & Ref & & Ref & \\
\hline Right & 0.44 & $0.37-0.51$ & 0.43 & $0.36-0.52$ \\
\hline \multicolumn{5}{|l|}{ Clinical $\mathrm{T}$ stage ${ }^{\mathrm{c}}$} \\
\hline$\leq \mathrm{T} 1$ & Ref & & Ref & \\
\hline $\mathrm{T} 2$ & 3.54 & $2.76-4.54$ & 2.86 & $2.21-3.69$ \\
\hline $\mathrm{T} 3$ & 8.23 & $6.36-10.64$ & 6.75 & $5.18-8.80$ \\
\hline $\mathrm{T} 4$ & 15.51 & $10.94-21.98$ & 14.74 & $10.20-21.30$ \\
\hline Unknown & 4.08 & $2.75-6.04$ & 3.78 & $2.52-5.65$ \\
\hline \multicolumn{5}{|l|}{ Postoperative histology } \\
\hline Adenocarcinoma & Ref & & Ref & \\
\hline Squamous cell carcinoma & 4.12 & $3.44-4.93$ & 3.58 & $2.94-4.35$ \\
\hline Different $^{\mathrm{d}}$ & 1.52 & $1.13-2.06$ & 1.49 & $1.09-2.03$ \\
\hline
\end{tabular}

${ }^{\wedge} O R$ odds ratio, $C I$ confidence interval. A confidence interval excluding 1.00 indicates statistical significance

*Univariable, **multivariable

${ }^{a}$ Forced expiratory volume in $1 \mathrm{~s}$, percentage of expected

${ }^{\mathrm{b}}$ Diffuse lung capacity for oxygen, percentage of expected

${ }^{c}$ According to TNM-7 staging

${ }^{\mathrm{d}}$ Adenosquamous or large cell carcinoma

(mortality: OR $0.28,95 \%$ CI $0.05-1.45$, complicated course: OR 1.42, 95\% CI 0.40-5.05).

When considering all anatomical resections, there were significantly more patients with a postoperative complicated course in the three hospitals with more pneumonectomies (430 patients included) compared to the three hospitals with less pneumonectomies (557 patients included) than expected (OR 1.51, 95\% CI 1.05-2.19), after adjustment for relevant factors. There was no significant difference in postoperative mortality between these groups (OR 0.66, 95\% CI 0.28-1.54). 
Fig. 1 Funnel plot of betweenhospital variation in the use of pneumonectomy (2012-2016).

* O/E ratio: observed number of pneumonectomies divided by expected $^{\wedge}$ number of pneumonectomies. $* * O=E$ : the observed number equals the expected $^{\wedge}$ number of pneumonectomies. ${ }^{\wedge}$ Expected number of pneumonectomies per hospital based on hospital population characteristics (age, gender, cardiac and pulmonary comorbidity, side of

malignancy, clinical $\mathrm{T}$ stage, histopathology). Number of hospitals included $N=51$; number of patients included $n=7885$

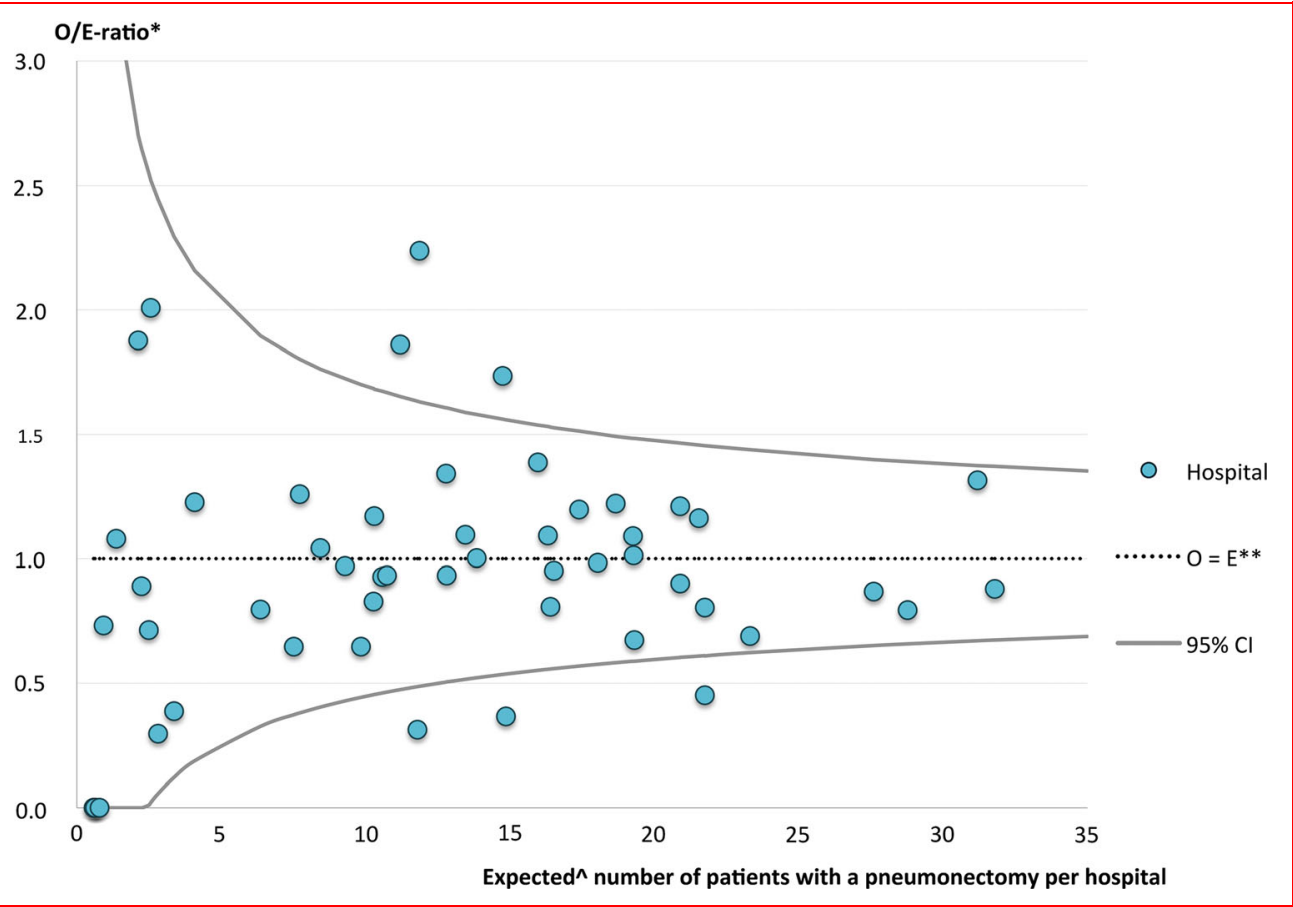

Fig. 2 Scatter of P/S ratio* per hospital (2015-2016). *P/S ratio: number of pneumonectomies divided by number of sleeve resections per hospitals. Number of hospitals included $\mathrm{N}=42$; number of patients included $n=3790$

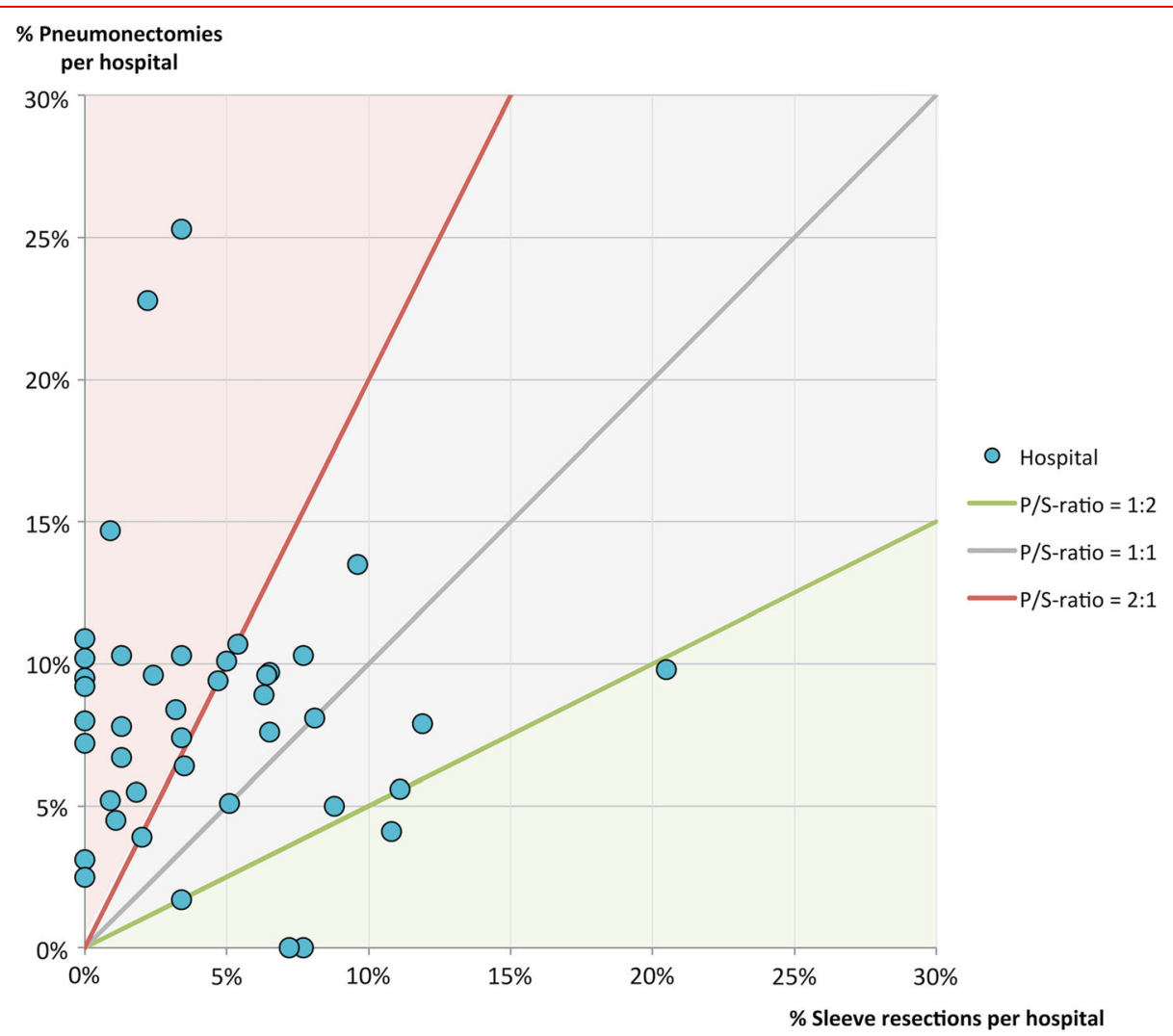

From 2015 on, the DLCA-S contains information on sleeve resections. Subgroup analysis of resections between 2015 and 2016 showed wide variation in the pneumonectomy/sleeve resection ratio per hospital (Fig. 2). Eight hospitals performed no sleeve resection and up to $10.9 \%$ pneumonectomies. Two hospitals performed up to $7.7 \%$ sleeve resections and no pneumonectomies. 


\section{Comment}

This study is the first to report on both the national practice of pneumonectomy use and between-hospital variation by using Dutch nationwide registry data with center-specific information. Considerable between-hospital variation exists in the use of pneumonectomy for primary lung cancer in the Netherlands, even after adjustment for patient and disease characteristics.

\section{National variation}

In the current study, age, gender, cardiac and pulmonary comorbidities, tumor side, cT and histopathology were individual factors significantly associated with receiving a pneumonectomy. This is in line with previous studies, although the current study was the first to perform multivariable analyses [20, 21].

Between-hospital variation in pneumonectomy use in the Netherlands ranged from 0.0 to $25.3 \%$ (IQR: $5.5-10.1 \%)$. After adjustment for relevant factors, out of 51 hospitals, three hospitals performed significantly more and three significantly less pneumonectomies than expected based on predetermined patient/disease characteristics.

The proportion of severe postoperative complications was higher in the hospitals with significantly more pneumonectomies. There were no significant differences in postoperative mortality between the hospitals performing significantly more or significantly less pneumonectomies. However, pneumonectomy-related mortality and morbidity often express beyond the 30-day follow-up period $[1,8,10]$; thus, the outcomes reported in this study could be an underestimation.

The existence of between-hospital variation suggests that for individual patients the risk to receive a pneumonectomy, and its related morbidity, could depend on the hospital of choice. Pneumonectomies may be performed on lower thresholds in some hospitals, whereas others might perform sleeve lobectomies or no resection at all. Of course, one cannot simply assume that every sleeve resection is an averted pneumonectomy; however, the varying pneumonectomy/sleeve resection ratio does indicate that considerations per hospital vary. The proportion of pneumonectomies per hospital might also be influenced by the availability of alternative treatment strategies (e.g., chemo(radiotherapy) instead of surgery in T3/T4 tumors) or the preference of local multidisciplinary teams. Whether referral patterns or patient preferences influence the between-hospital differences could not be studied, since these data were not available from the DLCA-S. However, the fact that all six 'outlying' hospitals are medium-sized non-academic centers lowers the presumption of referral bias. In addition, potential bias was reduced by the casemix adjustment.

Unlike previous literature and the intuitive expectation that centers with a high pneumonectomy proportion would have better postoperative outcomes after a pneumonectomy, in this study no significant differences were observed in postoperative mortality and complicated course after a pneumonectomy performed in hospitals with a high versus low pneumonectomy proportion. This could suggest that high pneumonectomy proportions are rather an expression of varying treatment considerations per hospital than the result of referral to expertise centers. It can also be hypothesized that higher pneumonectomy percentages could be the result of more unplanned pneumonectomies due to intra-operative complications or a preoperatively underestimated tumor stage.

\section{Centralization of care}

In the past years, the number of hospitals providing surgical lung cancer care declined from 79 in 2005 to 43 hospitals in 2015 , signifying a $45 \%$ reduction [14, 22]. In the current study, there were 42 hospitals performing sleeve resections or pneumonectomies in 2015-2016. In this period, there were 30 hospitals performing between 1 and 10 sleeve resections and 28 hospitals performing between 1 and 10 pneumonectomies. Although a considerable centralization has been achieved, a further centralization might be necessary for the technically difficult or high-risk procedures as sleeve resections or pneumonectomies, since it is know that volume could influence surgical outcomes [23].

\section{International comparison}

The pneumonectomy proportion in the Netherlands (7.8\%) is lower than in most European countries (7.4-19.6\%), but higher than in the USA (4.8\%) (supplemental Table 1) [6-12]. A pneumonectomy proportion as high as $34.6 \%$ was reported by a regional cohort study from the Netherlands (1984-1992) [24]. More historical English and Danish registry data show a significant decrease in pneumonectomy proportion over time [6,7]. The study by Jakobsen et al. reports a national decrease from $23 \%$ to $11 \%$ (2000-2007) [6]. Postoperative mortality after pneumonectomy in the Netherlands $(7.1 \%)$ is similar to other European countries (5.9-8.0\%), but slightly higher than in the USA (4.9\%).

Making these international comparisons, one should keep in mind that studied populations differ. The Society of Thoracic Surgeons General Thoracic Surgery Database (GTSD) and the European Society of Thoracic Surgeons GTSD data included resections not only for primary lung 
cancer (87.0-94.5\%), but also for metastasis (1.9-4.5\%) and benign diseases (3.6-3.8\%) [20]. Besides, the Dutch population is older and less frequently treated with induction therapy, and tumor (pT) stage is less frequently missing, though comparable to European and American populations $[9,20]$.

Although there are previous studies reporting on the national pneumonectomy proportion and regional variation, this study is the first to report between-hospital variation. What also distinguishes the current study from previous ones is the way data are collected and used, influencing data quality, completeness and analytic possibilities. Data for this study were collected using a national prospective audit system [13, 25]. The audit itself is designed and maintained by clinicians, therefore including clinically most relevant information. Clinicians receive weekly updated feedback information, thereby enhancing data quality. Participation in the audit is incorporated in the professional quality system, and registered data are regularly checked by external data verification, thereby stimulating unbiased information. This is in contrast to registries with a more voluntary nature or a pure retrospectively registration.

\section{Study limitations}

A limitation of the DLCA-S is that it does not provide information on non-operated patients; thus, resection rates cannot be calculated, nor could the indication for (not) operating be studied. In accordance with English and Danish registry data [6, 7], another study showed an increasing lung cancer resection rate in the Netherlands [26]. This, together with stable pneumonectomy rates and population characteristics during the existence of the DLCA-S, suggests that the relatively low pneumonectomy proportion in the Netherlands is not due to risk-averse behavior. The DLCA-S data 2012-2016 did not provide information whether a tumor is centrally located or extends beyond fissures. This is registered from 2017 onwards. Proxy information used in this study is tumor ( $\mathrm{T}$ ) stage and histopathology, since squamous cell carcinoma is more often centrally located [27]. Also, the DLCA-S does not provide information on the percentage of aborted procedures stratified by the extent of surgery. A probably underestimated percentage of 1.2 of all patients undergoing surgery for NSCLC in the DLCA-S with no resection in the end is reported previously [5].

Due to differences in definitions (e.g., mortality) and applied in- and exclusion criteria, it is challenging to generate true international comparisons. Consensus on key data items therefore should be a shared objective.

\section{Future perspectives}

Awareness among caregivers on pneumonectomy use in practice can increase by providing benchmarked information regarding the pneumonectomy proportion per hospital in indicator format.

National data can be used to evaluate current clinical practice and trigger improvement initiatives. In colorectal cancer surgery for example, data from the clinical audit led to a modification of the national guideline adjustment and led to remarkable changes in clinical practice [28].

In addition, indicator results and between-hospital variation can be used to support a more solid quality of care discussion. Adjustment for patient/disease characteristics can place this information in context.

The DLCA-S scientific committee will work toward providing caregivers with this information. Since a pneumonectomy remains necessary to obtain complete oncologic resection in certain cases, a percentage of 0.0 pneumonectomies is not aspired. What the ideal 'target' pneumonectomy proportion would be is not yet clear. This will be subject of debate for the DLCA-S scientific committee and affiliated professional associations. Another point of discussion will be whether further concentration of high-risk procedures into expertise centers (with expertise in sleeve resections, high volume and optimal postoperative care) may be beneficial. Organizing this optimal care, equally accessible to all patients, is a combined responsibility of healthcare government and caregivers.

\section{Conclusions}

This study demonstrates that there is a considerable nationwide between-hospital variation in pneumonectomy use in surgical lung cancer treatment, even after adjustment for patient and disease characteristics. Variation could be the result of varying treatment considerations or unplanned pneumonectomies. Nationwide registries and the development of specifically focused pneumonectomy indicators could be improvement tools to further optimize surgical lung cancer care.

Acknowledgements The authors thank all surgeons, registrars, physician assistants and administrative nurses for data registration in the DLCA-S.

\section{Compliance with ethical standards}

Conflict of interest None declared for any of the authors.

Informed consent Not required for this study type under Dutch law. 
Funding This research did not receive any specific grant from funding agencies in the public, commercial, or not-for-profit sectors.

Open Access This article is distributed under the terms of the Creative Commons Attribution 4.0 International License (http://crea tivecommons.org/licenses/by/4.0/), which permits unrestricted use, distribution, and reproduction in any medium, provided you give appropriate credit to the original author(s) and the source, provide a link to the Creative Commons license, and indicate if changes were made.

\section{References}

1. Kopec SE, Irwin RS, Umali-Torres CB, Balikian JP, Conlan AA (1998) The postpneumonectomy state. Chest 114:1158-1184. https://doi.org/10.1378/chest.114.4.1158

2. Falcoz PE, Conti M, Brouchet L, Chocron S, Puyraveau M, Mercier $M$ et al (2007) The thoracic surgery scoring system (thoracoscore): risk model for in-hospital death in 15,183 patients requiring thoracic surgery. $\mathbf{J}$ Thorac Cardiovasc Surg 133:325-332. https://doi.org/10.1016/j.jtcvs.2006.09.020

3. Brunelli A, Salati M, Rocco G, Varela G, Van Raemdonck D, Decaluwe $\mathrm{H}$ et al (2017) European risk models for morbidity (EuroLung1) and mortality (EuroLung2) to predict outcome following anatomic lung resections: an analysis from the European Society of Thoracic Surgeons database. Eur J Cardio-Thorac Surg 51:1212. https://doi.org/10.1093/ejcts/ezx155

4. Fernandez FG, Kosinski AS, Burfeind W, Decamp MM, Seder C, Marshall B et al (2016) STS lung cancer resection risk model: higher quality data and superior outcomes. Ann Thorac Surg 102:370-377. https://doi.org/10.1016/j.athoracsur.2016.02.098. STS

5. Beck N, Hoeijmakers F, van der Willik EM, Heineman DJ, Braun J, Tollenaar RAEM et al (2018) National comparison of hospital performances in lung cancer surgery: the role of casemix adjustment. Ann Thorac Surg. https://doi.org/10.1016/j.athor acsur.2018.02.074

6. Jakobsen E, Palshof T, Østerlind K, Pilegaard H (2009) Data from a national lung cancer registry contributes to improve outcome and quality of surgery: Danish results. Eur J Cardio-Thorac Surg 35:348-352. https://doi.org/10.1016/j.ejcts.2008.09.026

7. Riaz SP, Linklater KM, Page R, Peake MD, Møller H, Lüchtenborg M (2012) Recent trends in resection rates among nonsmall cell lung cancer patients in England. Thorax 67:811-814. https://doi.org/10.1136/thoraxjnl-2012-201768

8. Powell HA, Tata LJ, Baldwin DR, Stanley RA, Khakwani A, Hubbard RB (2013) Early mortality after surgical resection for lung cancer: an analysis of the English National Lung cancer audit. Thorax 68:826-834. https://doi.org/10.1136/thoraxjnl2012-203123

9. Thomas PA, Berbis J, Baste JM, Le Pimpec-Barthes F, Tronc F, Falcoz PE et al (2015) Pneumonectomy for lung cancer: contemporary national early morbidity and mortality outcomes. J Thorac Cardiovasc Surg 149:73-82. https://doi.org/10.1016/j. jtcvs.2014.09.063

10. Green A, Hauge J, Iachina M, Jakobsen E (2016) The mortality after surgery in primary lung cancer: results from the Danish Lung Cancer Registry. Eur J Cardio-Thoracic Surg 49:589-594. https://doi.org/10.1093/ejcts/ezv107

11. Morgant MC, Pagès PB, Orsini $\mathrm{B}$, Falcoz PE, Thomas PA, Le Pimpec Barthes $\mathrm{F}$ et al (2015) Time trends in surgery for lung cancer in France from 2005 to 2012: a nationwide study. Eur
Respir J 46:1131-1139. https://doi.org/10.1183/13993003.003542015

12. Seder CW, Raymond DP, Wright CD, Gaissert HA, Chang AC, Clinton S et al (2017) The society of thoracic surgeons general thoracic surgery database 2017 update on outcomes and quality. Ann Thorac Surg 103:1378-1383. https://doi.org/10.1016/j.athor acsur.2017.02.073

13. Beck N, Hoeijmakers F, Wiegman EM, Smit JM, Schramel FM, Steup WH et al (2018) Lessons learned from the Dutch Institute for Clinical Auditing: the Dutch model for quality assurance in lung cancer treatment. J Thorac Dis. https://doi.org/10.21037/jtd. 2018.04.56

14. Ten Berge MG, Beck N, Heineman DJ, Damhuis RAM, Steup WH, van Huijstee PJ et al (2018) Dutch lung surgery audit: a national audit comprising lung and thoracic surgery patients. Ann Thorac Surg. https://doi.org/10.1016/j.athoracsur.2018.03.049

15. Dutch Institute for Clinical Auditing; verification project group. Data verification in the DLCA-S: Final report. 2016

16. Multidisciplinary workinggroup on thoracic cancer. Dutch guideline for diagnosis and treatment of Non Small-Cell Lung Cancer 2015

17. Bernard A, Ferrand L, Hagry O, Benoit L, Cheynel N, Favre J-P (2000) Identification of prognostic factors determining risk groups for lung resection. Ann Thorac Surg 70:1161-1167. https://doi.org/10.1016/S0003-4975(00)01853-1

18. Spiegelhalter DJ (2005) Funnel plots for comparing institutional performance. Stat Med 24:1185-1202. https://doi.org/10.1002/ sim. 1970

19. Fischer C, Lingsma H, Hardwick R, Cromwell DA, Steyerberg E, Groene O (2016) Risk adjustment models for short-term outcomes after surgical resection for oesophagogastric cancer. $\mathrm{Br} \mathbf{J}$ Surg 103:105-116. https://doi.org/10.1002/bjs.9968

20. Seder CW, Salati M, Kozower BD, Wright CD, Falcoz PE, Brunelli A et al (2016) Variation in pulmonary resection practices between the society of thoracic surgeons and the European society of thoracic surgeons general thoracic surgery databases. Ann Thorac Surg 101:2077-2084. https://doi.org/10.1016/j.athor acsur.2015.12.073

21. Pagès PB, Mordant P, Renaud S, Brouchet L, Thomas PA, Dahan $M$ et al (2017) Sleeve lobectomy may provide better outcomes than pneumonectomy for non-small cell lung cancer. A decade in a nationwide study. J Thorac Cardiovasc Surg 153:184-195. https://doi.org/10.1016/j.jtcvs.2016.09.060

22. Damhuis RA, Maat AP, Plaisier PW (2015) Performance indicators for lung cancer surgery in the Netherlands. Eur J Cardiothorac Surg 47:897-903. https://doi.org/10.1093/ejcts/ezu329 (discussion 903-4)

23. von Meyenfeldt EM, Gooiker GA, van Gijn W, Post PN, van de Velde CJ, Tollenaar RA et al (2012) The relationship between volume or surgeon specialty and outcome in the surgical treatment of lung cancer: a systematic review and meta-analysis. J Thorac Oncol 7:1170-1178. https://doi.org/10.1097/JTO. 0b013e318257cc45

24. Damhuis RAM, Schütte PR (1996) Resection rates and postoperative mortality in 7,899 patients with lung cancer. Eur Respir J 9:7-10. https://doi.org/10.1183/09031936.96.09010007

25. Hoeijmakers F, Beck N, Wouters MWJM, Prins HA, Steup WH (2018) National quality registries: how to improve the quality of data? J Thorac Dis. https://doi.org/10.21037/jtd.2018.04.146

26. Wouters MWJM, Siesling S, Jansen-Landheer ML, Elferink MAG, Belderbos J, Coebergh JW et al (2010) Variation in treatment and outcome in patients with non-small cell lung cancer by region, hospital type and volume in the Netherlands. Eur $\mathrm{J}$ Surg Oncol 36:S83-S92. https://doi.org/10.1016/j.ejso.2010.06. 020 
27. Rosado-de-Christenson ML, Frazier AA, Stocker JT, Templeton PA (1993) From the archives of the AFIP. Extralobar sequestration: radiologic-pathologic correlation. Radiographics 13:425-441. https://doi.org/10.1148/radiographics.13.2.8460228

28. Gietelink L, van Groningen J, Tollenaar RAEM, Wouters MWJM, Marijnen CAM, Wouters MWJM et al (2017) Changes in nationwide use of preoperative radiotherapy for rectal cancer after revision of the national colorectal cancer guideline. Eur $\mathbf{J}$ Surg Oncol 43:1297-1303. https://doi.org/10.1016/j.ejso.2016. 12.019

Publisher's Note Springer Nature remains neutral with regard to jurisdictional claims in published maps and institutional affiliations. 